\title{
Plasma depletion layer: its dependence on solar wind conditions and the Earth dipole tilt
}

\author{
Y. L. Wang ${ }^{1}$, J. Raeder ${ }^{2}$, and C.T. Russell ${ }^{3}$ \\ ${ }^{1}$ Los Alamos National Laboratory, Los Alamos, USA \\ ${ }^{2}$ University of New Hampshire, Durham, USA \\ ${ }^{3}$ University of California, Los Angeles, USA
}

Received: 26 November 2003 - Revised: 25 September 2004 - Accepted: 3 October 2004 - Published: 22 December 2004

\begin{abstract}
The plasma depletion layer (PDL) is a layer on the sunward side of the magnetopause with lower plasma density and higher magnetic field compared to their corresponding upstream magnetosheath values. It is believed that the PDL is controlled jointly by conditions in the solar wind plasma and the interplanetary magnetic field (IMF). In this study, we extend our former model PDL studies by systematically investigating the dependence of the PDL and the slow mode front on solar wind conditions using global MHD simulations. We first point out the difficulties for the depletion factor method and the plasma $\beta$ method for defining the outer boundary of the plasma depletion layer. We propose to use the N/B ratio to define the PDL outer boundary, which can give the best description of flux tube depletion. We find a strong dependence of the magnetosheath environment on the solar wind magnetosonic Mach number. A difference between the stagnation point and the magnetopause derived from the open-closed magnetic field boundary is found. We also find a strong and complex dependence of the PDL and the slow mode front on the IMF $B_{z}$. A density structure right inside the subsolar magnetopause for higher IMF $B_{z}$ might be responsible for some of this dependence. Both the IMF tilt and clock angles are found to have little influence on the magnetosheath and the PDL structures. However, the IMF geometry has a much stronger influence on the slow mode fronts in the magnetosheath. Finally, the Earth dipole tilt is found to play a minor role for the magnetosheath geometry and the PDL along the Sun-Earth line. A complex slow mode front geometry is found for cases with different Earth dipole tilts. Comparisons between our results with those from some former studies are conducted, and consistencies and inconsistencies are found.
\end{abstract}

Key words. Magnetospheric physics (magnetosheath, solar wind-magnetosphere interactions) - Space plasma physics (numerical simulation studies)

Correspondence to: Y. L. Wang

(ywang@lanl.gov)

\section{Introduction}

The plasma depletion layer (PDL) is a layer on the sunward side of the magnetopause with lower plasma density and higher magnetic field compared to their corresponding upstream magnetosheath values. As an important layer between the solar wind and the Earth's magnetosphere, the PDL has been found in some former studies to be controlled by the conditions in the solar wind (e.g. Farrugia et al., 1997; Siscoe et al., 2002; Wang et al., 2003, 2004b). In this paper we present a systematic study of the properties of the PDL for different solar wind conditions using global MHD simulations. Denton and Lyon (2000) have studied the effects of pressure anisotropy on the magnetosheath structure using a 2-D MHD model with anisotropic pressure and a flux surface magnetopause. They found that the exact form of the parallel pressure gradient force may not be crucial for the global dynamics of the PDL, except that the anisotropy leads to a larger bow shock standoff distance compared to the isotropic case. Their results also imply that the effects of pressure anisotropy may be even less for a 3-D system than for a 2-D system. Thus, our use of the isotropic MHD code appears to be appropriate.

Farrugia et al. (1997) studied three examples of lowlatitude ISEE 2 passes through the dayside magnetosheath near noon on 3 December 1979, 5 October 1979, and 11 November 1979, and they found that the properties of the plasma depletion layer depend on the solar wind Alfvén Mach number. Siscoe et al. (2002) summarized four important MHD effects in the magnetopause boundary layer and the magnetosheath that cannot be produced by gasdynamic models, including the PDL. They found a clear dependence of the PDL thickness on the IMF clock angle based on their global model results. Wang et al. (2003) compared their global MHD model results, using IMP 8 and ACE solar wind plasma and IMF observations as a driver, with Wind in-situ observations for two PDL events on 12 January 1996 and 1 January 1999. They found good visual consistency between PDL observations and their model results. Meanwhile, the 
Table 1. Model input parameters for different model runs in this PDL dependence study.

\begin{tabular}{lc}
\hline Model input parameters (in GSE) & Values \\
\hline$V_{x}(\mathrm{~km} / \mathrm{s})$ & $-450,-600,-750$ \\
$V_{y}(\mathrm{~km} / \mathrm{s})$ & 0 \\
$V_{z}(\mathrm{~km} / \mathrm{s})$ & 0 \\
$B_{x}(\mathrm{nT})$ & $0,7,14$ \\
$B_{y}(\mathrm{nT})$ & 0 \\
$B_{z}(\mathrm{nT})$ & $2,7,14,21$ \\
$\mathrm{~N}\left(\mathrm{~cm}^{-3}\right)$ & 6 \\
$\mathrm{~T}_{\mathrm{p}}=\mathrm{T}_{\mathrm{e}}(\mathrm{eV})$ & 10 \\
$\mathrm{IMF}$ tilt angle $\left(^{\circ}\right)$ & $0,45,63$ \\
$\mathrm{IMF}$ clock angle $\left(^{\circ}\right)$ & $0,15,30,45$ \\
Earth dipole tilt angle $\left(^{\circ}\right)$ & $0,15,30$ \\
\hline
\end{tabular}

average model departures, which are defined as the average separations between model results and observations, were found to be generally smaller than the standard deviations of observations in their study. They concluded that an MHD description can produce the plasma depletion features in the magnetosheath. Furthermore, they showed that the PDL is stable for stable solar wind conditions and small magnetosheath variations observed by single spacecraft were temporal, rather than spatial, variations during these two events. Wang et al. (2004b) went further to address the role of the slow mode waves for the formation of the PDL, and they confirmed the existence of the slow mode front in the magnetosheath for certain, but not all, stable solar wind conditions. Their results showed a clear dependence of the slow mode front on the IMF $B_{z}$. Although they concluded that the slow mode waves have little effect on the magnetosheath flow and field structures, including the plasma depletion layer, they did not exclude the possibility of a much larger role of such waves for the formation of the PDL for more turbulent magnetosheath conditions.

Until now, however, still no systematic study has been conducted for the dependence of the PDL on various solar wind parameters. Experimentally this is very difficult because of the scarcity of observations. This is mostly because satellites that have their apogee or perigee near the magnetopause distance, for example, Geotail, do not traverse the PDL, but rather skim along the magnetopause. From such measurements it is generally not even possible to determine if one has observed the PDL. On the other hand, satellites that traverse the magnetosheath rapidly provide data that allow one to identify the PDL. However, such orbits, for example, those of Wind, provide magnetosheath traverses at a much lower rate. Further, Wind's orbit is controlled and it penetrates the magnetosheath far from the subsolar point where the most important physics of the PDL occurs. Together with the requirement of concurrent observations of stable solar wind conditions, northward IMF preferred, leads to very few PDL observations.
An alternate approach is to use models to investigate and document the dependence of the PDL on solar wind conditions. Such an approach would be similar to the one taken by Spreiter et al. (1966) for the basic properties of the magnetosheath. Spreiter's model has proven extremely useful for many subsequent studies. However, his model is gasdynamic and thus does not include the plasma depletion layer. It is an approximation to the real magnetosheath environment because of its lack of the magnetic force and the use of a rigid magnetopause. Contemporary demands require a more thorough verification and understanding of the results from Spreiter's model. With the introduction of MHD and a self-consistent model, significantly more free parameters enter the problem compared to a study based on a gasdynamic model. At a minimum, the IMF strength and orientation need to be considered in addition to the gasdynamic parameters.

In this paper, we first introduce the parameters for the model runs that we have conducted for this study. Then we discuss the definition of the PDL outer boundary, which itself is a challenge for the PDL study. After that, we show the dependence of the PDL on the solar wind magnetosonic Mach number, the IMF $B_{z}$, and the IMF tilt and clock angles. Because of the special role of the slow mode waves in both observational and theoretical studies of the PDL formation (Zwan and Wolf, 1976; Song et al., 1990a, 1992; Southwood and Kivelson, 1992, 1995), we also address the dependence of the slow mode front on solar wind conditions. The results can help us better understand the wave environment in the magnetosheath, especially during turbulent solar wind conditions when the slow mode waves could play a much more important role to control the flow and field in the magnetosheath. Since the Earth dipole tilt also affects the coupling between the Earth's magnetosphere and the solar wind, we also address the dependence of the PDL and the slow mode front on it, which has seldom been done before. Finally, we summarize the results in this study.

\section{Model runs}

The Raeder global geospace model is used in this study. This model solves resistive MHD equations with isotropic pressure in a large, three-dimensional volume surrounding the Earth, such that the entire interaction region between the solar wind and the magnetosphere, as well as the ionosphere, is included (Raeder, 2003). Specifically, the simulation domain comprises the bow shock, the magnetopause, and the magnetotail up to several hundred $R_{E}$ from the Earth. The NOAA Coupled Thermosphere Ionosphere Model (CTIM) is included to handle the coupling between the magnetosphere and the ionosphere (Fuller-Rowell et al., 1996; Raeder et al., $2001 b$ ). In this study, however, we only use uniform ionosphere conductivity of 5 Siemens because the ionosphere is likely to have little influence on the magnetosheath flows.

In order to study the dependence of the PDL on solar wind conditions and the Earth dipole tilt, we make a series of global model runs with a solar wind density of $\mathrm{N}=6 \mathrm{~cm}^{-3}$, 

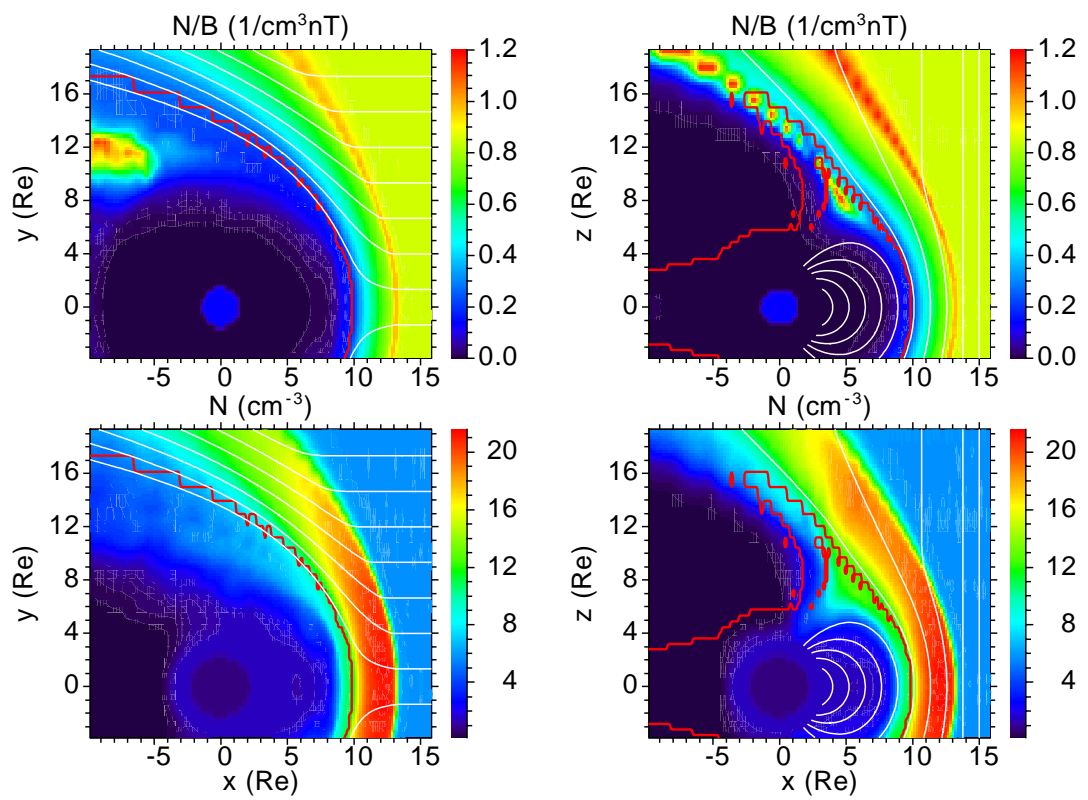

Fig. 1. The utility of the N/B ratio to mark the outer boundary of the plasma depletion layer. Shown here are the global model results with the baseline solar wind conditions as input. The left panels show the results in the GSE $\mathrm{z}=0$ plane, and the right panels show the results in the GSE $y=0$ plane. The N/B values are shown as the background on the top panels, and the plasma density values are shown as the background on the bottom panels. The open-closed magnetic field boundary is shown as a red zigzag curve in each of the panels. The flow lines are shown as white smooth curves in the left panels, and magnetic field lines are shown as white smooth curves in the right panels.

an interplanetary magnetic field $\boldsymbol{B}=(0,0,7) \mathrm{nT}$, a solar wind velocity $\boldsymbol{V}=(-450,0,0) \mathrm{km} / \mathrm{s}$, and a solar wind temperature of $\mathrm{T}_{\mathrm{p}}=\mathrm{T}_{\mathrm{e}}=10 \mathrm{eV}$ as the baseline solar wind conditions. The input parameters for the model runs in this study are shown in Table 1 . Those values in Table 1 that have only a single number are not varied in the parameter tests.

In each parameter test, the global geospace model is run for three hours in physical time, which is sufficient for the model to overcome its start-up effects. The results shown in this paper are obtained at the final time of each run, which reflect stable subsolar magnetopause and magnetosheath configurations.

\section{The definition of the PDL boundary}

The plasma depletion layer is not a very well-defined structure in the magnetosheath. Although it is more or less sharply bounded by the magnetopause on the downstream side, it usually has no sharp boundary on the upstream side but a rather gradual change in the magnetic field magnitude and the plasma density. Defining the upstream boundary is therefore difficult and any method must be to some extent arbitrary.

Currently, there are two most commonly used methods to define the PDL outer boundary: the depletion factor method and the plasma $\beta$ method. In the depletion factor method, the depletion factor, $\lambda$, is defined as the ratio between the plasma density just after the bow shock and that on the mag- netopause. This method was first introduced by Zwan and Wolf (1976) and it has been used in some other PDL studies (e.g. Siscoe et al., 2002; Song and Russell, 2002). Zwan and Wolf (1976) found $\lambda$ to be $3-4$ at the stagnation point and they defined the thickness of the depletion layer to be the distance from the stagnation point to the half post-bow shock density point $(\lambda=2)$. The PDLs with $\lambda$ larger than 2 have been observed (e.g. Crooker et al., 1979; Paschmann et al., 1993; Phan et al., 1994; Wang et al., 2003). However, most numerical model studies obtained smaller $\lambda$ values (e.g. $\mathrm{Wu}$, 1992; Lyon, 1994). Siscoe et al. (2002) obtained a $\lambda$ of $\sim 10$, which is not only much higher than most other numerical model results, but also much larger than the usually observed $\lambda$ values. Later in this paper, we show that $\lambda$ is controlled by solar wind conditions. The plasma $\beta$ method has also been used by some authors (e.g. Farrugia et al., 1997) to define the PDL outer boundary. The plasma $\beta$ is defined as the ratio between the plasma pressure and the magnetic pressure, which reveals the relative contribution of the plasma and magnetic field in controlling plasma motion. When $\beta<1$, the magnetic field is playing a more important role. When $\beta>1$, plasma is more important. Usually from the bow shock to the magnetopause, the $\beta$ value decreases and the magnetic field is playing a more and more important role in controlling plasma motion. It is convenient to use a particular $\beta$ value to define the PDL outer edge. Farrugia et al. (1997) believed that this definition captures the physical meaning of the plasma depletion layer in which the magnetic force dominates over the plasma pressure force. Usually $\beta=1$ was used to define the 




Fig. 2. Some parameters along the SunEarth line in Fig. 1. The four panels, from top to bottom, show the flow speed, the magnetic field magnitude, the plasma density, and the N/B ratio. The magnetopause is defined as the location where the magnetosheath flow speed is close to zero. The shaded region marks the PDL and the dashed line marks the bow shock.
PDL outer boundary. However, sometimes they used $\beta=0.5$ for a more restrictive definition.

The depletion factor method defines the PDL outer boundary in a more descriptive way, and the plasma $\beta$ method defines the PDL outer boundary in a more physical way. Although these two methods are very easy to use in practice, they have major difficulties. For the depletion factor method, it misses the earlier drop of the plasma density in the magnetosheath, which includes important physics that distinguishes the PDL from the other part of the magnetosheath. For the plasma $\beta$ method, it assumes that the plasma $\beta$ is the controlling factor of the PDL. However, this assertion lacks sound observational and theoretical support. To be specific, if the solar wind Mach number is low, $\beta<1$ could occur in all of the magnetosheath, which makes such a definition of the PDL outer boundary impossible. Wang et al. (2003) showed that the PDL formation is a three-dimensional MHD effect with complex interactions between the plasma pressure gradient force and the magnetic force in the magnetosheath. In the PDL and the other magnetosheath region, both forces play important roles in shaping the magnetosheath. This may not be fully reflected by a single $\beta$ value. Specifically, plasma motion along a flux tube, which is crucial for flux tube depletion, is only controlled by the plasma pressure gradient force along that flux tube and has nothing to do with the plasma $\beta$ value. Another important difficulty for both of these methods is that they are fairly arbitrary and, in practice, various values have been used to define the outer boundary for different purposes.

Because the physics of the PDL is more related to the depletion of plasma along flux tube, e.g. flux tube depletion, than purely plasma density decrease, it could be a good idea to use a quantity best fit to describe flux tube depletion to mark the outer boundary of a plasma depletion layer. One such quantity is the N/B ratio. Figure 1 shows the global model results with the baseline solar wind conditions as input. The left panels show the results in the GSE $z=0$ plane, and the right panels show the results in the GSE $y=0$ plane. The N/B ratio is shown as the background in the top panels, and the plasma density is shown as the background in the bottom panels. A layer with decreased plasma density is shown in each of the bottom panels of Fig. 1. We also see a layer on the magnetopause with decreased N/B ratio, which means flux tube depletion. Some parameters along the Sun-Earth line in Fig. 1 are shown in Fig. 2. The four panels from top to bottom show the flow speed, the magnetic field magnitude, the plasma density, and the N/B ratio. The magnetopause is defined as the location where the magnetosheath flow speed is close to zero. In Fig. 2, there is a weak decrease of the N/B ratio from the bow shock toward the magnetopause. A steeper N/B ratio decrease is seen closer to the magnetopause (the shaded region in Fig. 2) which differentiates a layer from the other part of the magnetosheath. Thus, we can use the N/B ratio to define the outer boundary of the PDL by analyzing the different trends of it from the bow shock to the magnetopause. More specifically, in the depletion layer, there is a steeper N/B ratio decrease toward the magnetopause, while in the other part of the magnetosheath the N/B ratio decrease is weaker. We can define the boundary between the two different N/B ratio trends as the outer boundary of the PDL. In this way, we can not only capture the sharpest change for flux tube depletion, but also avoid 

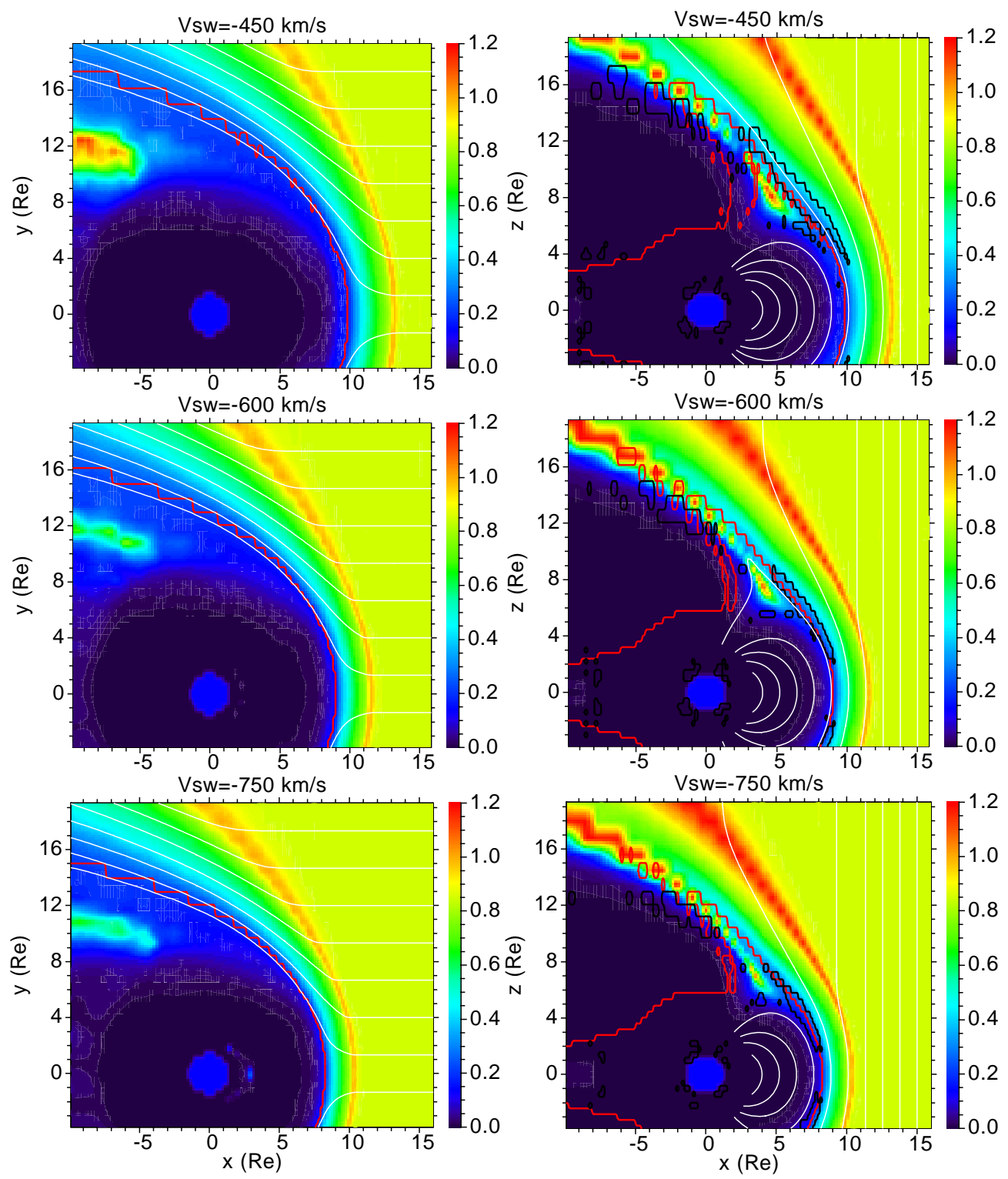

Fig. 3. The dependence of the PDL and the slow mode front on the solar wind $\mathrm{M}_{\mathrm{MS}}$ using different solar wind velocities: $V_{x}=-450 \mathrm{~km} / \mathrm{s}$ $\left(\mathrm{M}_{\mathrm{MS}}=5.3\right),-600 \mathrm{~km} / \mathrm{s}\left(\mathrm{M}_{\mathrm{MS}}=7.1\right)$, and $-750 \mathrm{~km} / \mathrm{s}\left(\mathrm{M}_{\mathrm{MS}}=8.8\right)$ as model inputs. The other solar wind input parameters are: $V_{y}=V_{z}=0 \mathrm{~km} / \mathrm{s}$, $\boldsymbol{B}=(0,0,7) \mathrm{nT}, \mathrm{N}=6 \mathrm{~cm}^{-3}$, and $\mathrm{T}_{\mathrm{p}}=\mathrm{T}_{\mathrm{e}}=10 \mathrm{eV}$. The left panels show the results in the $\mathrm{GSE} \mathrm{z}=0$ plane, and the right panels show the results in the GSE $y=0$ plane. The N/B ratio is shown as the background and the open-closed magnetic field boundary is shown as a red zigzag curve in each of the panels. Flow lines are shown as white smooth curves in the left panels, and magnetic field lines are shown as white smooth curves in the right panels. The boundary between the regions where the slow mode group velocity can and cannot overcome the flow velocity are shown as black zigzag curves in the right panels. The slow mode fronts are the segments of these black curves where plasma flows into the closed black curve regions (Wang et al., 2004b).

using arbitrary parameters like the depletion factor method and the plasma $\beta$ method. Although this task can usually be easily done by visual analysis, there are many difficulties in finding an algorithm to automatically determine such an outer boundary of the PDL. For example, in certain latitudes and longitudes, the PDL structure becomes very flat, and the N/B ratio trend is difficult to tell even for visual evaluation.

\section{The effects of the solar wind magnetosonic Mach number}

The magnetosonic Mach number is defined as: $\mathrm{M}_{\mathrm{MS}}=\mathrm{V} / \sqrt{\mathrm{V}_{\mathrm{A}}^{2}+\mathrm{C}_{\mathrm{S}}^{2}}$, here $\mathrm{V}$ is the plasma flow speed, $V_{A}$ the Alfvén speed, and $C_{s}$ the sound speed. Figure 3 shows the dependence of the PDL and the slow mode front on the solar wind $\mathrm{M}_{\mathrm{MS}}$ using three different solar wind velocities $V_{x}=-450 \mathrm{~km} / \mathrm{s} \quad\left(\mathrm{M}_{\mathrm{MS}}=5.3\right), \quad-600 \mathrm{~km} / \mathrm{s}$ 


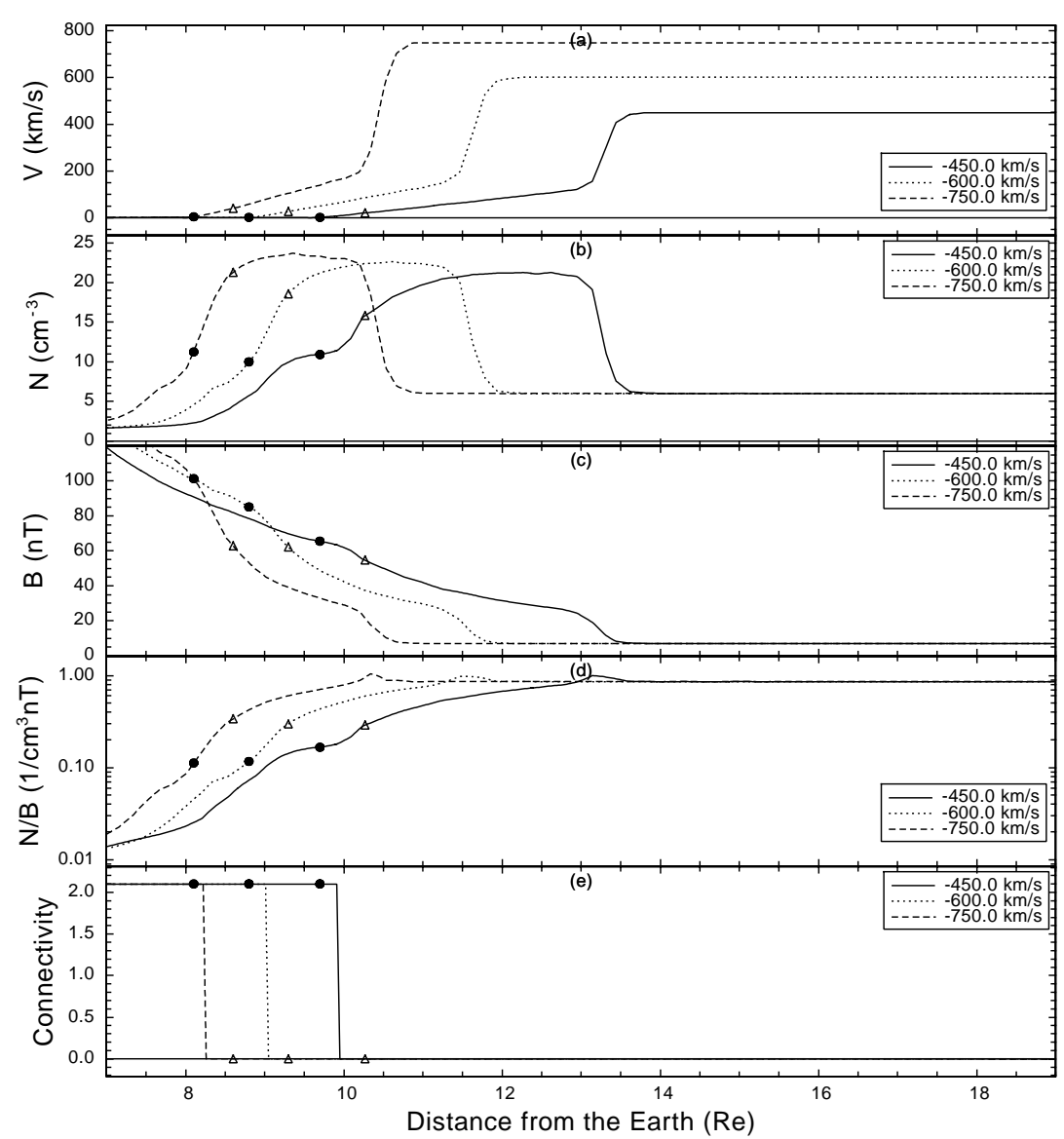

Fig. 4. The flow speed, the plasma density, the magnetic field magnitude, the N/B ratio, and the field line connectivity along the Sun-Earth line for the three runs with different solar wind $\mathrm{M}_{\mathrm{MS}}$ in Fig. 3. The magnetosphere is on the left side and the solar wind is on the right side of each panel. The black dot on each line stands for the stagnation point on that line where the magnetosheath flow speed reaches close to zero. The blank triangle on each line stands for the outer boundary of the PDL which is defined by the trend change of the N/B ratio along that line. The field line connectivity is defined as the number of the ends of a field line that connect to the Earth.
$\left(\mathrm{M}_{\mathrm{MS}}=7.1\right)$, and $-750 \mathrm{~km} / \mathrm{s}\left(\mathrm{M}_{\mathrm{MS}}=8.8\right)$ as model inputs. The other solar wind input parameters are: $V_{y}=V_{z}=0 \mathrm{~km} / \mathrm{s}$, $\boldsymbol{B}=(0,0,7) \mathrm{nT}, \mathrm{N}=6 \mathrm{~cm}^{-3}$, and $\mathrm{T}_{\mathrm{p}}=\mathrm{T}_{\mathrm{e}}=10 \mathrm{eV}$. The left panels of the figure show the results in the GSE $\mathrm{z}=0$ plane, and the right panels show the results in the GSE $y=0$ plane. The N/B ratio is shown as the background and the open-closed magnetic field boundary is shown as a red zigzag curve in each of the panels. Flow lines are shown as white smooth curves in the left panels, and magnetic field lines are shown as white smooth curves in the right panels. The boundary between the regions where the slow mode group velocity can and cannot overcome the flow velocity are shown as black zigzag curves in the right panels. The slow mode fronts are the segments of the black curves where plasma flows into the closed black curve regions (Wang et al., 2004b). In Fig. 3, the magnetopause and the magnetosheath are more strongly compressed for higher solar wind velocity (higher $\mathrm{M}_{\mathrm{MS}}$ ) than for lower solar wind velocity (lower $\mathrm{M}_{\mathrm{MS}}$ ). This is because higher solar wind velocity corresponds to larger dynamic pressure. There is very little difference for the density structures in the magnetosheath, except that these structures are more compressed for higher solar wind $\mathrm{M}_{\mathrm{MS}}$. However, there are obvious differences for the slow mode front, which is shown in the right panels of Fig. 3 . More specifically, with the increasing solar wind $\mathrm{M}_{\mathrm{MS}}$, the slow mode fronts are pushed closer toward the open-closed magnetic field boundary and they also move closer to the GSE $\mathrm{z}=0$ plane.

Figure 4 shows some important parameters along the SunEarth line for the three runs with different solar wind $\mathrm{M}_{\mathrm{MS}}$. The magnetosphere is on the left side and the solar wind is on the right side of each panel. The black dot on each line stands for the stagnation point on that line where the magnetosheath flow speed reaches close to zero. For this definition of the stagnation point we follow Siscoe et al. (2002). The blank triangle on each line stands for the outer boundary of the PDL which is defined by the trend change of the N/B ratio along that line in the magnetosheath. With the increasing solar wind velocity, the stagnation point moves toward the Earth, and the thickness of the plasma depletion layer decreases.

Song et al. (1999) pointed out the distinction between the stagnation point, as we use here, and the magnetopause defined as the last closed field line surface, which is often used in model studies. Siscoe et al. (2002) found the similar results in their global model study for the northward IMF case. In panel (e) of Fig. 4, we show the field line connectivity, which is defined as the number of connections of a field line with the Earth. We see that the magnetopause, as defined by the last closed magnetic field line, does extend further out into the magnetosheath than the stagnation point. This is consistent with both Song et al. (1999) and Siscoe et al. (2002), 

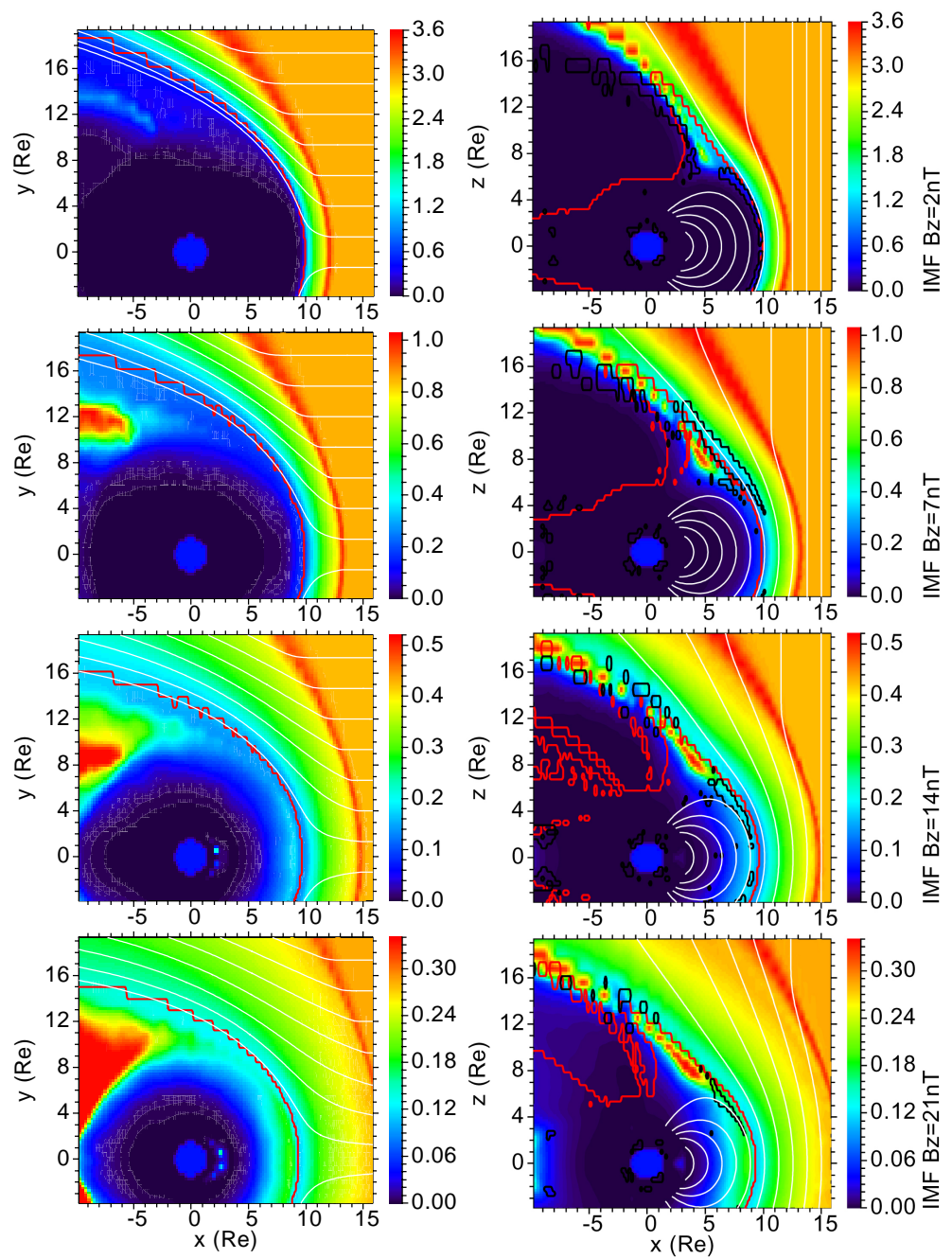

Fig. 5. The dependence of the PDL and the slow mode front on the IMF $B_{z}: 2,7,14$, and $21 \mathrm{nT}$. The other solar wind input parameters are: $B_{x}=B_{y}=0 \mathrm{nT}, \boldsymbol{V}=(-450,0,0) \mathrm{km} / \mathrm{s}, \mathrm{N}=6 \mathrm{~cm}^{-3}$, and $\mathrm{T}_{\mathrm{p}}=\mathrm{T}_{\mathrm{e}}=10 \mathrm{eV}$. The other formats of this figure are the same as those in Fig. 3.

and it is likely a result of the reconnection process poleward of the cusp. The poleward cusp reconnection usually occurs during northward IMF when antiparallel or quasi-antiparallel configuration forms between magnetosheath and lobe field lines (e.g. Dungey, 1961). A magnetosheath field line can become closed field line through this poleward reconnection (Song and Russell, 1992). However, the field line can still keep some magnetosheath flow speed. This can explain the more outward extension of the open-closed magnetic field boundary than the stagnation point.

Historically, the depletion factor has been used as an important parameter for characterizing the PDL and comparing between different model results. The depletion factors from some other studies and this study (using baseline solar wind conditions) for more or less similar solar wind conditions are shown in Table 2. The results of Wu (1992) and Lyon (1994) lead to very small depletion factors. While the depletion factor from Siscoe et al. (2002) is much higher than all the other results. Our depletion factor is intermediate com-
Table 2. Comparison between the depletion factors from different studies.

\begin{tabular}{lc}
\hline Sources & Depletion factor, $\lambda$ \\
\hline Zwan and Wolf (1976) & $3-4$ \\
Wu (1992) & 1.3 \\
Lyon (1994) & 1.2 \\
Siscoe et al. (2002) & 10 \\
This study & 1.95 \\
\hline
\end{tabular}

pared to the other model results, and it is closer to Zwan and Wolf (1976)'s result.

Table 3 shows the dependence of some important parameters on the solar wind $\mathrm{M}_{\mathrm{MS}}$. Here $\mathrm{M}_{\mathrm{A}}$ is the solar wind Alfvén Mach number, $\mathrm{H}_{\mathrm{PDL}}$ is the PDL thickness, $\mathrm{L}_{\mathrm{MP}}$ is the distance between the magnetopause and the Earth, $\mathrm{L}_{\mathrm{BS}}$ is the distance between the bow shock and the Earth, $\mathrm{H}_{\mathrm{MS}}$ 
Table 3. The dependence of some important parameters along the Sun-Earth line on the solar wind $\mathrm{M}_{\mathrm{MS}}$.

\begin{tabular}{ccccccccc}
\hline $\mathrm{M}_{\mathrm{MS}}$ & $\mathrm{V}_{\mathrm{sw}}(\mathrm{km} / \mathrm{s})$ & $\mathrm{M}_{\mathrm{A}}$ & $\beta$ & $\lambda$ & $\mathrm{H}_{\mathrm{PDL}}\left(R_{E}\right)$ & $\mathrm{L}_{\mathrm{MP}}\left(R_{E}\right)$ & $\mathrm{L}_{\mathrm{BS}}\left(R_{E}\right)$ & $\mathrm{H}_{\mathrm{MS}}\left(R_{E}\right)$ \\
\hline 5.3 & -450 & 7.2 & 1.0 & 1.95 & 0.60 & 9.7 & 13.1 & 3.4 \\
7.1 & -600 & 9.6 & 1.0 & 2.33 & 0.57 & 8.8 & 11.4 & 2.6 \\
8.8 & -750 & 12.0 & 1.0 & 2.05 & 0.50 & 8.1 & 10.2 & 2.1 \\
\hline
\end{tabular}

Table 4. The dependence of some important parameters on the IMF $B_{z}$ along the Sun-Earth line.

\begin{tabular}{ccccccccc}
\hline IMF $B_{z}(\mathrm{nT})$ & $\mathrm{M}_{\mathrm{A}}$ & $\mathrm{M}_{\mathrm{MS}}$ & $\beta$ & $\lambda$ & $\mathrm{H}_{\mathrm{PDL}}\left(R_{E}\right)$ & $\mathrm{L}_{\mathrm{MP}}\left(R_{E}\right)$ & $\mathrm{L}_{\mathrm{BS}}\left(R_{E}\right)$ & $\mathrm{H}_{\mathrm{MS}}\left(R_{E}\right)$ \\
\hline 2 & 25.3 & 7.5 & 12.1 & 1.31 & 0.15 & 9.9 & 12.0 & 2.1 \\
7 & 7.2 & 5.3 & 1.0 & 1.91 & 0.57 & 9.7 & 13.1 & 3.4 \\
14 & 3.6 & 3.3 & 0.2 & 1.48 & 1.00 & 9.4 & 14.5 & 5.1 \\
21 & 2.4 & 2.3 & 0.1 & 1.05 & 1.05 & 9.1 & 16.5 & 7.4 \\
\hline
\end{tabular}

is the thickness of the magnetosheath, and $\beta$ is for the solar wind which includes both electron and ion pressure in its calculation. With increasing solar wind $\mathrm{M}_{\mathrm{MS}}$, both the bow shock and the magnetopause are more compressed toward the Earth, and the magnetosheath thickness along the Sun-Earth line decreases too. As a result, the PDL is also squeezed. The depletion factor varies with the solar wind $\mathrm{M}_{\mathrm{MS}}$ but not monotonically. When the solar wind $\mathrm{M}_{\mathrm{MS}}$ is high, the depletion factor becomes larger than 2. Although there is no slow mode front along the Sun-Earth line in all the three cases (see the right panels of Fig. 3), the PDL exists (see Fig. 4). This further confirms our conclusion in Wang et al. (2004b) that the slow mode front is not a necessary condition for the PDL formation. Similar results are obtained for many other cases later in the paper, which will not be discussed.

\section{The effects of the IMF $B_{z}$}

Figure 5 shows the dependence of the PDL and the slow mode front on the IMF $B_{z}: 2,7,14$, and $21 \mathrm{nT}$. The other solar wind input parameters are: $B_{x}=B_{y}=0 \mathrm{nT}$, $\boldsymbol{V}=(-450,0,0) \mathrm{km} / \mathrm{s}, \mathrm{N}=6 \mathrm{~cm}^{-3}$, and $\mathrm{T}_{\mathrm{p}}=\mathrm{T}_{\mathrm{e}}=10 \mathrm{eV}$. In the figure, the larger the IMF $B_{z}$ is, the stronger the magnetopause is compressed toward the Earth (because of the enhanced solar wind magnetic pressure), and the farther the bow shock extends toward the solar wind (because of the enhanced fast mode wave velocity, and the reduction of the $\mathrm{M}_{\mathrm{MS}}$ ), and the thicker the magnetosheath. In Fig. 5, with increasing IMF $B_{z}$, a larger density structure starts to occur near the subsolar point right inside the magnetopause. This density structure extends in both the $\mathrm{z}=0$ plane and the $\mathrm{y}=0$ plane and its peak density increases with the IMF $B_{z}$. This density structure is likely caused by the plasma flow from the polar reconnection. If so, the larger the IMF $B_{z}$, the stronger the polar reconnection to produce larger plasma density inside the subsolar magnetopause. Meanwhile, there is a strong dependence of the slow mode front on the IMF $B_{z}$, which is shown in the right panels of Fig. 5. For IMF $B_{z}=2 \mathrm{nT}$, the slow mode fronts occur inside the open-closed magnetic field boundary and it extends almost to the GSE $\mathrm{z}=0$ plane. This is very different from the results for IMF $B_{z}=7 \mathrm{nT}$ in which case the slow mode fronts are farther from the openclosed magnetic field boundary in the magnetosheath and farther from the GSE $\mathrm{z}=0$ plane. For IMF $B_{z}=14$ and $21 \mathrm{nT}$, the slow mode fronts return inside the open-closed magnetic field boundary and they become more compressed and closer to the GSE $\mathrm{z}=0$ plane compared to the IMF $B_{z}=7 \mathrm{nT}$ case.

Figure 6 shows the flow speed, the plasma density, the magnetic field magnitude, and the N/B ratio along the SunEarth line for the four model runs with different IMF $B_{z}$ in Fig. 5. There is a very strong dependence of the PDL structures on the IMF $B_{z}$. Specifically, there is no clear PDL structure for IMF $B_{z}=2 \mathrm{nT}$ (the plasma pressure is much larger than the magnetic pressure). In this case the plasma pressure is playing a crucial role and the plasma in the magnetosheath is acting more like the gasdynamic case which produces no plasma depletion. When IMF $B_{z}=7 \mathrm{nT}$, very clear PDL structure occurs. When the IMF $B_{z}$ is larger, the PDL structure becomes wider and more smoothed out. This is likely caused by the much stronger magnetic force than the plasma pressure gradient force in the magnetosheath, as well as the much more stretched out magnetosheath. The density peaks inside the subsolar magnetopause are larger for higher IMF $B_{z}$ cases and they are likely caused by the enhanced polar reconnection.

Some important parameters along the Sun-Earth line for different IMF $B_{z}$ are listed in Table 4. The depletion factor does not change linearly with the IMF $B_{z}$. Instead, it first increases then decreases with the IMF $B_{z}$. The later decrease of the depletion factor with the IMF $B_{z}$ can be explained by the enhanced polar reconnection which produces the density increase near the stagnation point. With increasing IMF $B_{z}$, the magnetopause is almost linearly pushed toward the 


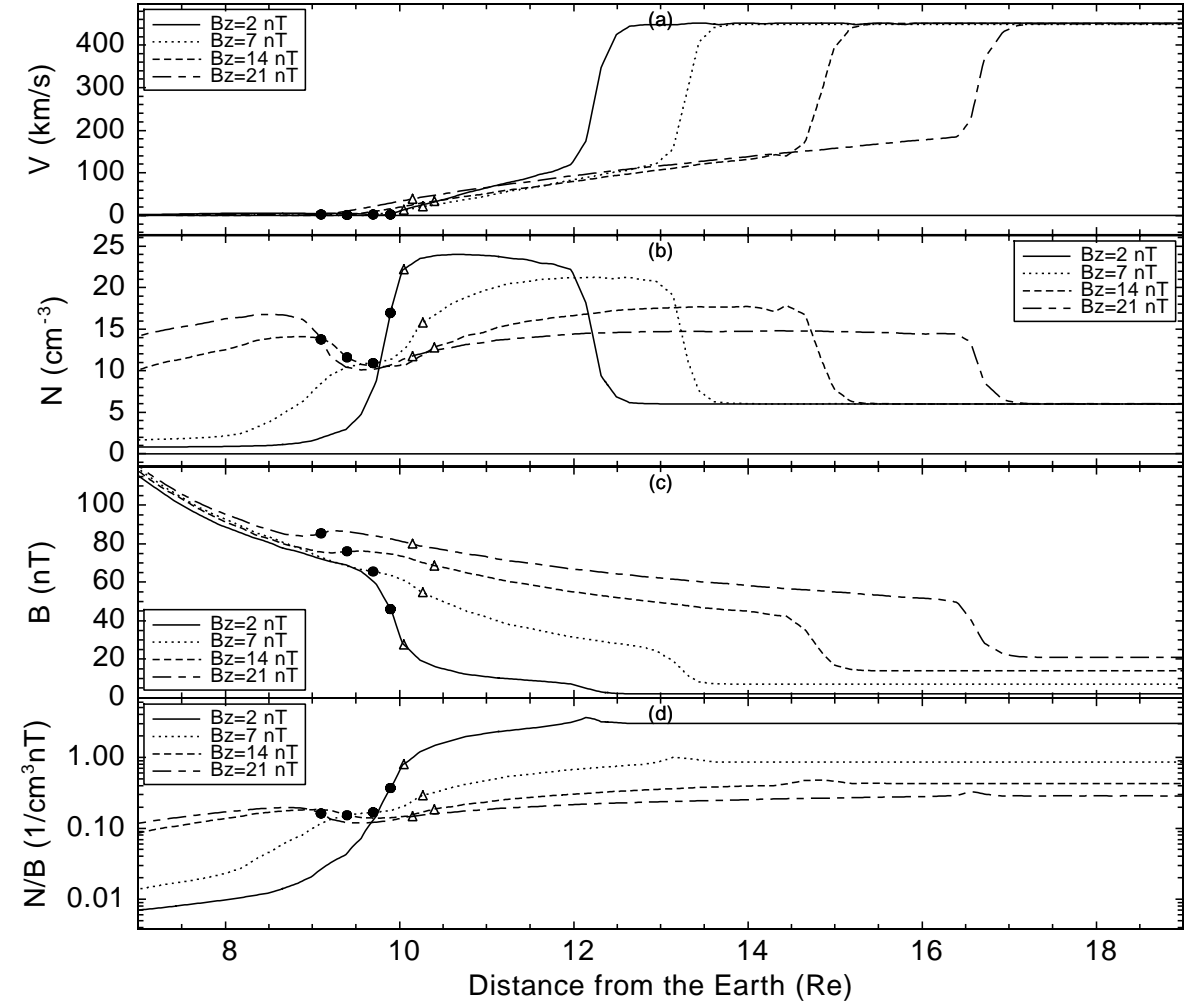

Fig. 6. The flow speed, the plasma density, the magnetic field magnitude, and the N/B ratio along the Sun-Earth line in Fig. 5. The other formats of this figure are the same as those in Fig. 4.
Earth, and the bow shock extends almost linearly into the solar wind. As a result, the thickness of the magnetosheath increases almost linearly from $\sim 2 R_{E}$ for IMF $B_{z}=2 \mathrm{nT}$ to $\sim 7 R_{E}$ for IMF $B_{z}=21 \mathrm{nT}$. Similarly, the PDL thickness increases monotonically with the IMF $B_{z}$.

\section{The effects of the IMF tilt angle}

Figure 7 shows the dependence of the PDL and the slow mode front on the IMF tilt angle: $0^{\circ}$ (IMF $B_{x}=0 \mathrm{nT}$ ), $45^{\circ}$ (IMF $B_{x}=7 \mathrm{nT}$ ), and $63^{\circ}$ (IMF $B_{x}=14 \mathrm{nT}$ ). The other solar wind input parameters are: IMF $B_{y}=0 \mathrm{nT}$ and $B_{z}=7 \mathrm{nT}$, $\boldsymbol{V}=(-450,0,0) \mathrm{km} / \mathrm{s}, \mathrm{N}=6 \mathrm{~cm}^{-3}$, and $\mathrm{T}_{\mathrm{p}}=\mathrm{T}_{\mathrm{e}}=10 \mathrm{eV}$. In the left panels of Fig. 7, there are different magnetosheath flow line structures, starting from the same locations in the solar wind, for different IMF tilt angles. This means that the plasma flow in the magnetosheath is to some extent controlled by the IMF tilt angle. Although the IMF orientation is different from case to case, there is only very little change for the locations of the magnetopause and the bow shock in the GSE $\mathrm{z}=0$ and $\mathrm{y}=0$ planes. In contrast to all the former cases, an asymmetry develops for the N/B ratio structure in the GSE $y=0$ plane. Specifically, the peak of the $\mathrm{N} / \mathrm{B}$ ratio inside the magnetosheath moves to the north of the GSE $\mathrm{z}=0$ plane for IMF tilt angle $=45^{\circ}$ and $63^{\circ}$ in the tests. This can be explained, referring to the magnetic field lines in the right panels of Fig. 7, by the fact that the plasma below the GSE $\mathrm{z}=0$ plane moves more along magnetic field, thus it can move easier. While the plasma to the north of the
GSE $\mathrm{z}=0$ plane moves more perpendicular to magnetic field, and it feels more magnetic pressure force generated by the piling up of the magnetic field on the magnetopause. As a result, the plasma density builds up more above the GSE $\mathrm{z}=0$ plane.

Similar to the N/B ratio asymmetry, the slow mode fronts also show a strong asymmetry for the cases with non zero IMF tilt angles. For the case with IMF tilt angle $=45^{\circ}$, a large slow mode front is seen below the GSE $\mathrm{z}=0$ plane and a very small slow mode front is seen above the plane. Meanwhile, the slow mode front below is closer to the GSE $\mathrm{z}=0$ plane than the one above. This slow mode front asymmetry increases with the IMF tilt angle. When IMF tilt angle $=63^{\circ}$, the slow mode fronts above and below the GSE $\mathrm{z}=0$ plane attach to each other to form a large slow mode front, whose lower part extends very far into the magnetosheath and is very close to the bow shock.

Figure 8 shows the flow speed, the plasma density, the magnetic field magnitude, and the N/B ratio along the SunEarth line in Fig. 7. There is very little difference for these cases with different IMF tilt angles. Specifically, the locations of the magnetopause and the bow shock do not change much for different IMF tilt angles. Meanwhile, the PDL shows a very similar pattern along the Sun-Earth line for the different cases in this study. The parameters along other neighboring radial lines show similar results, except a little shift of the bow shock location and a little change in the plasma depletion pattern on the magnetopause. By comparison, the effects of the IMF tilt angle (the IMF $B_{x}$ ) are much 

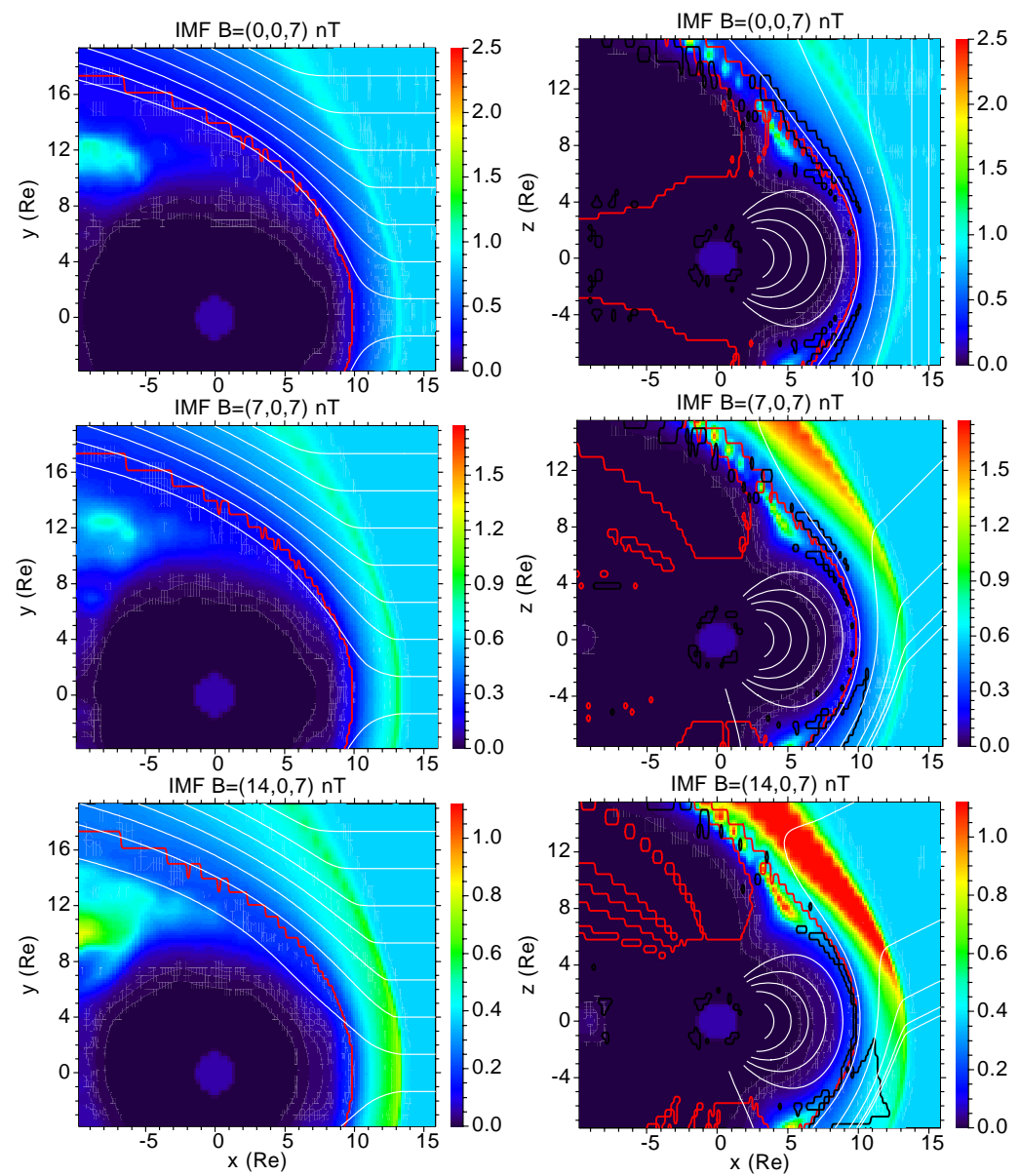

Fig. 7. The dependence of the PDL and the slow mode front on the IMF tilt angle. In these cases, we have the same IMF $B_{y}=0 \mathrm{nT}$ and $B_{z}=7 \mathrm{nT}$, but different IMF $B_{x}=0,7$, and $14 \mathrm{nT}$. The other solar wind input parameters are: $V=(-450,0,0) \mathrm{km} / \mathrm{s}, \mathrm{N}=6 \mathrm{~cm}-3$, and $\mathrm{T}_{\mathrm{p}}=\mathrm{T}_{\mathrm{e}}=10 \mathrm{eV}$. The other formats of this figure are the same as those in Fig. 3.

smaller than those of the IMF $B_{z}$. This result is consistent with some observations (e.g. Farrugia et al., 1997) and model results (e.g. Raeder et al., 2001a; Wang et al., 2003). Further, the IMF $B_{x}$ is not likely a major controlling factor for the PDL, at least near the Sun-Earth line.

Lee et al. (1991) simulated the enhanced plasma pressure and decreased magnetic field intensity in the magnetosheath, as observed by Song et al. (1990b), using a 2-D incompressible MHD simulation code. They found that, when there is a normal component of the IMF $\left(B_{x} \neq 0\right)$, the total magnetic field intensity tends to decrease in front of the depletion layer due to the bending of magnetic field lines, and the plasma pressure is enhanced in this region. On the other hand, when IMF $B_{x}=0$, this slow-mode structure is not present in the simulation and only the plasma depletion layer is observed. Our results have shown that, in cases with both IMF $B_{x}=0$ and $B_{x} \neq 0$, there is no structure with enhanced plasma density and decreased magnetic field. The difference between Lee et al. (1991)'s results and our results is likely caused by the different models used in the study. Lee et al. (1991) used a simplified model in their study. Since incompressibility is assumed in their simulation, actually no plasma density depletion can be obtained in the magnetosheath. Further, a 2-D simulation is also a limitation, which is unlikely to be able to give a reasonable description of the PDL formation as discussed in Wang et al. (2004a).

\section{The effects of the IMF clock angle}

Figure 9 shows the dependence of the PDL and the slow mode front on the IMF clock angle: $0^{\circ}, 15^{\circ}, 30^{\circ}$, and $45^{\circ}$ with the same IMF magnetic field magnitude $(7 \mathrm{nT})$. The other solar wind input parameters are: $\boldsymbol{V}=(-450,0,0) \mathrm{km} / \mathrm{s}$, $\mathrm{N}=6 \mathrm{~cm}^{-3}$, and $\mathrm{T}_{\mathrm{p}}=\mathrm{T}_{\mathrm{e}}=10 \mathrm{eV}$. In the figure, little influence of the IMF clock angle on the magnetosheath plasma structures, including the PDL, is seen in the GSE $\mathrm{z}=0$ and $\mathrm{y}=0$ planes. Specifically, there is little change for the locations of the bow shock, the magnetopause, and the magnetosheath. The slow mode front, however, is strongly influenced by the IMF clock angle. The slow mode front for $0^{\circ} \mathrm{IMF}$ clock angle has been discussed in former sections. For a $15^{\circ}$-IMF clock angle, the 


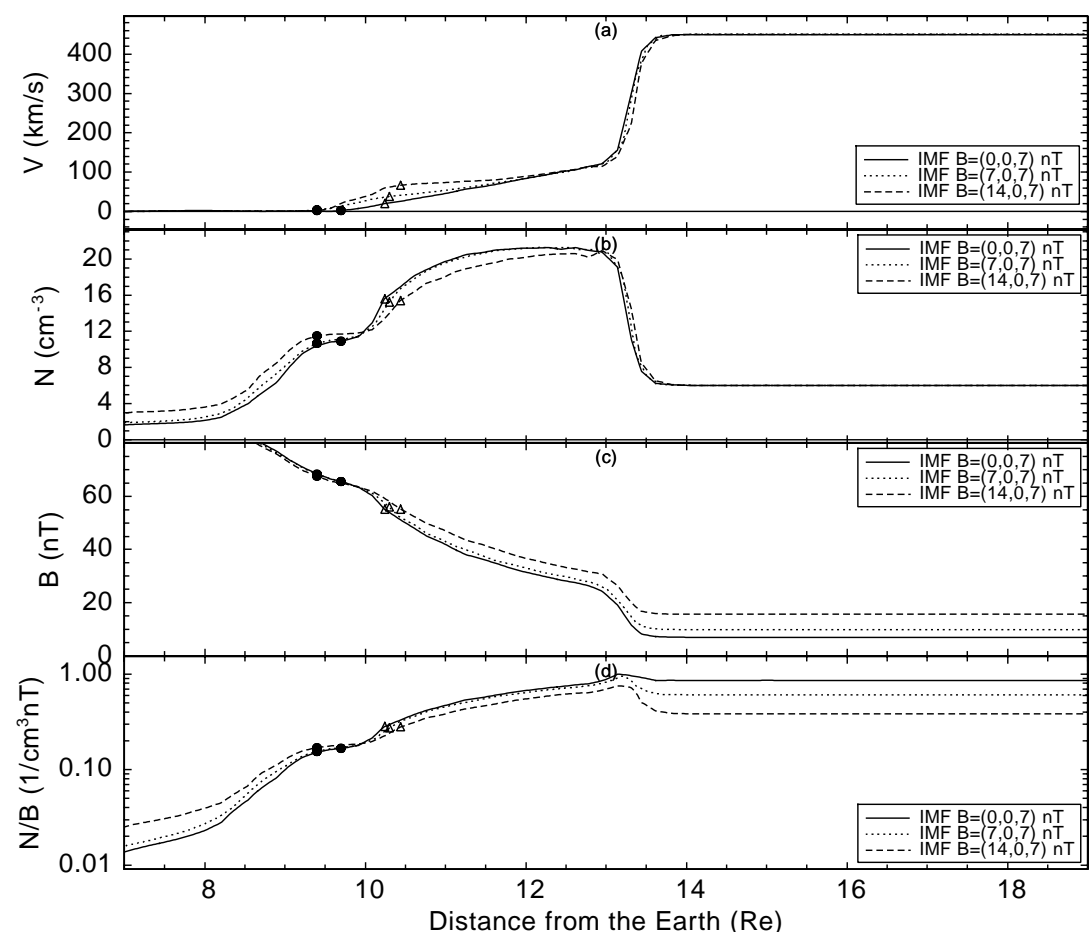

Fig. 8. The flow speed, the plasma density, the magnetic field magnitude, and the N/B ratio along the Sun-Earth line in Fig. 7. The other formats of this figure are the same as those in Fig. 4.

Table 5. The dependence of some important parameters on the IMF clock angle along the Sun-Earth line in Fig. 9.

\begin{tabular}{ccccccccc}
\hline Clock angle $\left(^{\circ}\right)$ & $\mathrm{M}_{\mathrm{A}}$ & $\mathrm{M}_{\mathrm{MS}}$ & $\beta$ & $\lambda$ & $\mathrm{H}_{\mathrm{PDL}}\left(R_{E}\right)$ & $\mathrm{L}_{\mathrm{MP}}\left(R_{E}\right)$ & $\mathrm{L}_{\mathrm{BS}}\left(R_{E}\right)$ & $\mathrm{H}_{\mathrm{MS}}\left(R_{E}\right)$ \\
\hline 0 & 7.2 & 5.3 & 1.0 & 1.95 & 0.57 & 9.7 & 13.1 & 3.4 \\
15 & 7.2 & 5.3 & 1.0 & 2.01 & 0.57 & 9.7 & 13.0 & 3.3 \\
30 & 7.2 & 5.3 & 1.0 & 2.08 & 0.80 & 9.7 & 13.0 & 3.3 \\
45 & 7.2 & 5.3 & 1.0 & 2.27 & 0.80 & 9.7 & 12.8 & 3.1 \\
\hline
\end{tabular}

slow mode front significantly shrinks in the $y=0$ plane. Further, for $30^{\circ}$ and $45^{\circ}$ clock angles, there is basically no slow mode front in the $\mathrm{y}=0$ plane in the magnetosheath.

Figure 10 shows the flow speed, the plasma density, the magnetic field magnitude, and the N/B ratio along the SunEarth line in Fig. 9. Very little difference is seen in the magnetosheath for these cases with different IMF clock angles, except that there are some little deviations between them at the bow shock and right before the stagnation point. Big differences are seen for the density immediately inside the subsolar magnetopause. Specifically, the smaller the IMF clock angle, the higher the plasma density inside the magnetopause. The plasma density inside the subsolar magnetopause is likely coming from the polar reconnection. If this is true, then the different density peak magnitudes inside the magnetopause reflect the reconnection rate for different IMF clock angles. The smaller the IMF clock angle, the stronger the polar reconnection, and the more solar wind plasma can go from the cusp to the subsolar magnetosphere. Table 5 shows the dependence of some important parameters on the IMF clock angle along the Sun-Earth line in Fig. 9. The de- pletion factor increases slightly with the IMF clock angle, although this dependence is much weaker than the depletion factor dependence on the solar wind $\mathrm{M}_{\mathrm{MS}}$ and the IMF $B_{z}$. Most of the small depletion factor increase is due to the little differences in the density structures near the stagnation point. Similarly, very little or no dependence is seen for the locations of the bow shock and the magnetopause. As a result, there is very little change in the thickness of the magnetosheath. Finally, there is a non monotonic dependence of the PDL thickness on the IMF clock angle.

Figure 11 shows the normalized plasma density along the stagnation streamline for different IMF conditions from Siscoe et al. (2002). Angles specify the clock angle of the IMF. The two vertical lines in each panel show where the velocity goes to zero (the stagnation point) and where the density drops to half its post-shock value. We see a large structural difference between the cases with $0^{\circ}$ and $45^{\circ}$ IMF clock angles. Specifically, the $0^{\circ}$ case has a long bottom extending into the magnetosphere, while the $45^{\circ}$ case has a sharp density cutoff following the density drop close to the magnetopause. As a result, the magnetopause density 

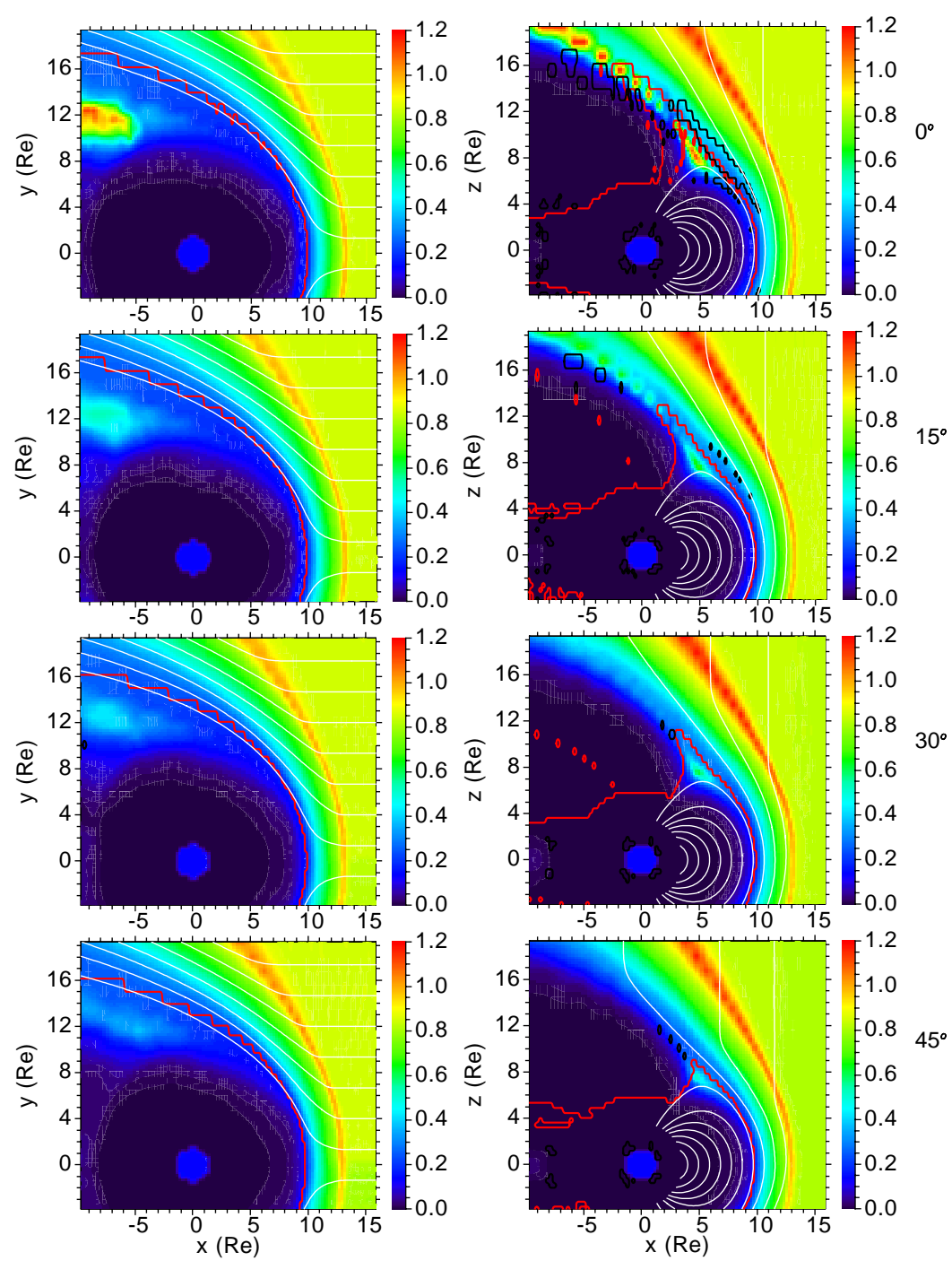

Fig. 9. The dependence of the PDL and the slow mode front on the IMF clock angle: $0^{\circ}, 15^{\circ}, 30^{\circ}$, and $45^{\circ}$ with the same IMF magnetic field magnitude $(7 \mathrm{nT})$. The other solar wind input parameters are: $V=(-450,0,0) \mathrm{km} / \mathrm{s}, \mathrm{N}=6 \mathrm{~cm}^{-3}$, and $\mathrm{T}_{\mathrm{p}}=\mathrm{T}_{\mathrm{e}}=10 \mathrm{eV}$. The other formats of this figure are the same as those in Fig. 3.

is close to zero for the $45^{\circ}$ case but a finite value $(\sim 0.5)$ for the $0^{\circ}$ case. Figure 12 shows the dependence of the depletion layer thickness obtained from Fig. 11 on the IMF clock angle. A value of $0.15 R_{E}$, representing the resolution of their simulations, has been subtracted from the solid line to obtain the dashed line. In both Figs. 11 and 12 there is a much stronger dependence of the PDL thickness on the IMF clock angle. Specifically, from $0^{\circ}$ to $45^{\circ}$ IMF clock angle, the thickness of the PDL varies from $\sim 0.43 R_{E}$ to $\sim 0.3 R_{E}$, which is very different from our results in both magnitude and trend. The solar wind parameters used in Siscoe et al. (2002) are: $\mathrm{V}=-350 \mathrm{~km} / \mathrm{s}, \mathrm{N}=5 \mathrm{~cm}^{-3}, \mathrm{~T}=20 \mathrm{eV}$, and $\mathrm{B}=5 \mathrm{nT}$, which are different from what we have used. However, the different solar wind inputs are not likely to cause the big differences between their results and ours. Siscoe et al. (2002) used the ISM model and we use the Raeder global geospace model in our PDL studies. Many settings in these two models are different, for example, numerical grids and numerical resistivity. Those different settings are likely to be responsible for the large differences between our results. The different methods to define the outer boundary of the PDL, the depletion factor method by Siscoe et al. (2002) and the N/B ratio method by us, are also likely to contribute to such differences. As a test of the accuracy of the Raeder global geospace model, we conducted case studies in Wang et al. (2003) and good consistency was obtained between our model results and spacecraft observations.

\section{The effects of the Earth dipole tilt}

The major difference between the Earth dipole tilt and the IMF tilt in the GSE $y=0$ plane is the solar wind flow direction 

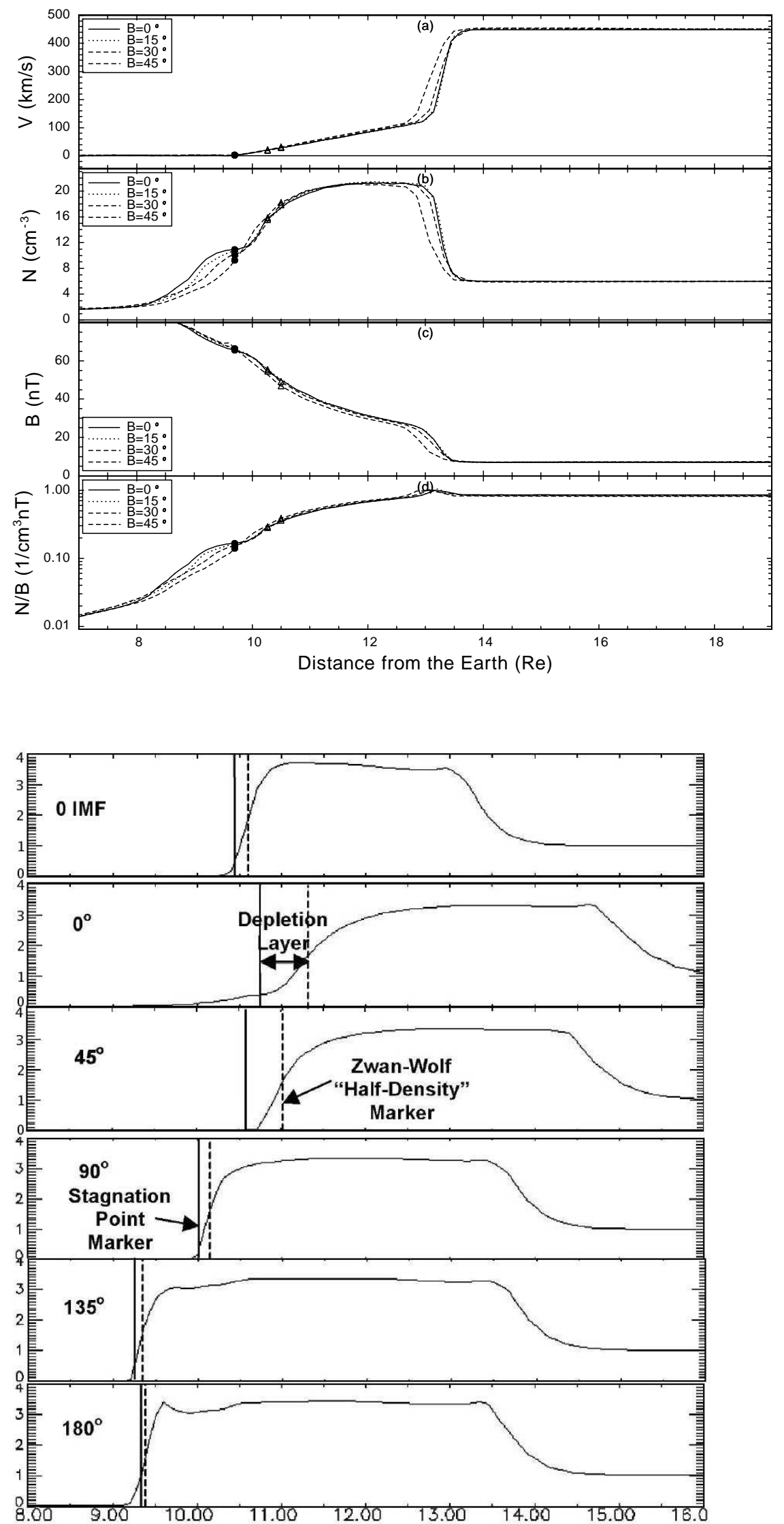

Fig. 10. The flow speed, the plasma density, the magnetic field magnitude, and the N/B ratio along the Sun-Earth line in Fig. 9. The other formats of this figure are the same as those in Fig. 4.

Fig. 11. Taken from Siscoe et al (2002). Normalized plasma density along the stagnation streamline for different IMF conditions. Angles specify the clock angle of the IMF. The two vertical lines in each panel show where the velocity goes to zero (the stagnation point) and where the density drops to half its post-shock value (to determine the thickness of the depletion layer by the Zwan and Wolf criterion). 


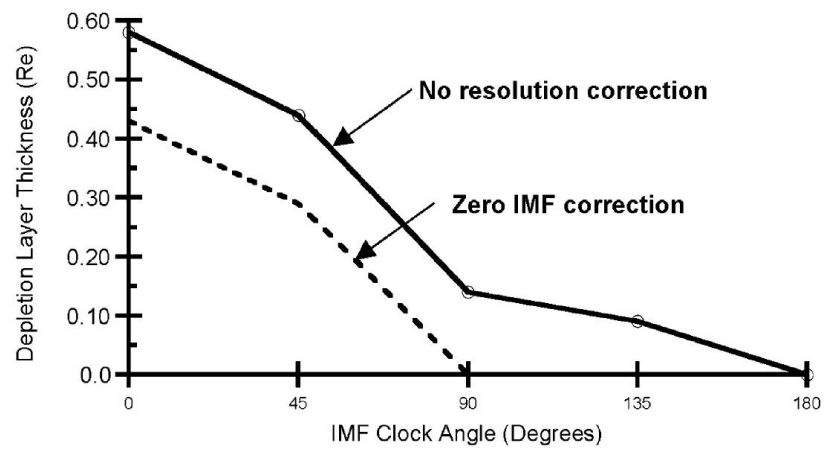

Fig. 12. Taken from Siscoe et al. (2002). Dependence of the thickness of the depletion layer, as defined by Zwan and Wolf (1976), obtained from Fig. 11 on the IMF clock angle. A value of $0.15 R_{E}$, representing the resolution of the process, has been subtracted from the solid line to obtain the dashed line.

relative to the Sun-Earth line. Thus, the effects of the Earth dipole tilt cannot be obtained using the results in Sect. 6 . Figure 13 shows the dependence of the PDL and the slow mode front on the Earth dipole tilt in the GSE $\mathrm{y}=0$ plane: $0^{\circ}$, $15^{\circ}$, and $30^{\circ}$. The baseline solar wind conditions are used as model input, which correspond to a solar wind $\mathrm{M}_{\mathrm{MS}}=5.3$. Similar to Fig. 9, there is little difference in the magnetosheath structures, including the locations of the bow shock and the magnetopause, for different Earth dipole tilts. Meanwhile, no strong N/B asymmetry is seen for the different cases. The strongest influenced by the Earth dipole tilt is the slow mode front. The slow mode front for $0^{\circ}$ Earth dipole tilt has been discussed earlier in the paper. For $15^{\circ}$ Earth dipole tilt, the slow mode fronts above and below the GSE $\mathrm{z}=0$ plane are connected to each other. The slow mode front structure is not symmetric, which is obviously caused by the non-zero Earth dipole tilt. There are segments of the slow mode front extending into the magnetosheath in both north and south of the GSE $\mathrm{z}=0$ plane. The major difference between them is that the north slow mode front is more detached from the center slow mode front, while the south slow mode front is closely connected with the center slow mode front, although there is a long bulge extending along the field line into the magnetosheath. The $30^{\circ}$ Earth dipole tilt case is similar to the former case, but with a more asymmetric slow mode front. The slow mode front north of the GSE $\mathrm{z}=0$ plane, which occurs for the $15^{\circ}$ Earth dipole tilt, almost disappears in this case. But there is little change for its southern counterpart.

Figure 14 shows the plasma $V_{z}$, the flow speed, the plasma density, the magnetic field magnitude, and the N/B ratio along the Sun-Earth line in Fig. 13. The black dot on each line marks the location of the magnetopause on that line which is defined by the close to zero flow velocity or the reverse of $V_{z}$. There are very distinct structures near the magnetopause for the flow speed, which are different from all the other cases that we have shown earlier. These differences are obviously connected with the Earth dipole tilt. If we follow the former definition of the magnetopause $(\mathrm{V}=0)$, the mag- netopause for the dipole tilt $=15^{\circ}$ and $30^{\circ}$ would be closer to the Earth. The reason for adding an extra panel in Fig. 14, $V_{z}$, is to show whether we should use the previous way to define the magnetopause. Panel (a) of Fig. 14 shows that the $V_{z}$ changes direction farther out from the point where the flow velocity reaches close to zero. We believe the downward flow closer to the Earth is likely the flow produced by the polar reconnection, which should be inside the magnetopause, while the upward flow farther from the Earth is the magnetosheath flow. Thus, it could be more accurate to define the magnetopause as the boundary between the upward and the downward flows. In panel (e), the N/B trend in the magnetosheath is very weak, which makes the definition of the PDL outer boundary very difficult. Here, we use the density trend in the magnetosheath as a complement to accomplish this task. In the figure, there is little difference for the parameters in the magnetosheath for the different Earth dipole tilt angles, except in the small region close to the magnetopause where the PDL occurs.

Table 6 shows the detailed dependence of some important parameters along the Sun-Earth line on the Earth dipole tilt. There is a large drop of the depletion factor when the dipole tilt angle increases from $0^{\circ}$ to $15^{\circ}$. A smaller depletion factor drop is seen when the dipole tilt angle changes from $15^{\circ}$ to $30^{\circ}$. In all the three cases with different Earth dipole tilt angles, little difference is seen for the locations of the magnetopause and the bow shock, as well as the thickness of the magnetosheath. Meanwhile, the PDL thickness only changes slightly.

\section{Discussion and conclusions}

The magnetosphere is driven by the solar wind through the coupling between them on the magnetopause. As an important structure on the magnetopause usually during northward IMF conditions, the plasma depletion layer clearly differentiates itself with lower plasma density and higher magnetic field than the upstream magnetosheath conditions. Such a boundary condition can undoubtedly affect the mass, momentum, and energy coupling between the solar wind and the magnetosphere. It has long been known that the coupling between the solar wind and the magnetosphere has a different degree of dependence on solar wind conditions. A more quantitative understanding of such a dependence is especially important for the space weather study which requires accurate representation of the Sun-Earth connection and magnetosphere dynamics. However, such a systematic and extensive study about the dependence of the PDL on different conditions has never been performed. This is an important incentive for this work. Because of the strong limitations of PDL observations and the oversimplifications of analytical models in describing the PDL, we have been using the Raeder global magnetosphere model in this study, which has proven to be able to describe the fundamental physics of the plasma depletion layer (Wang et al., 2003). 

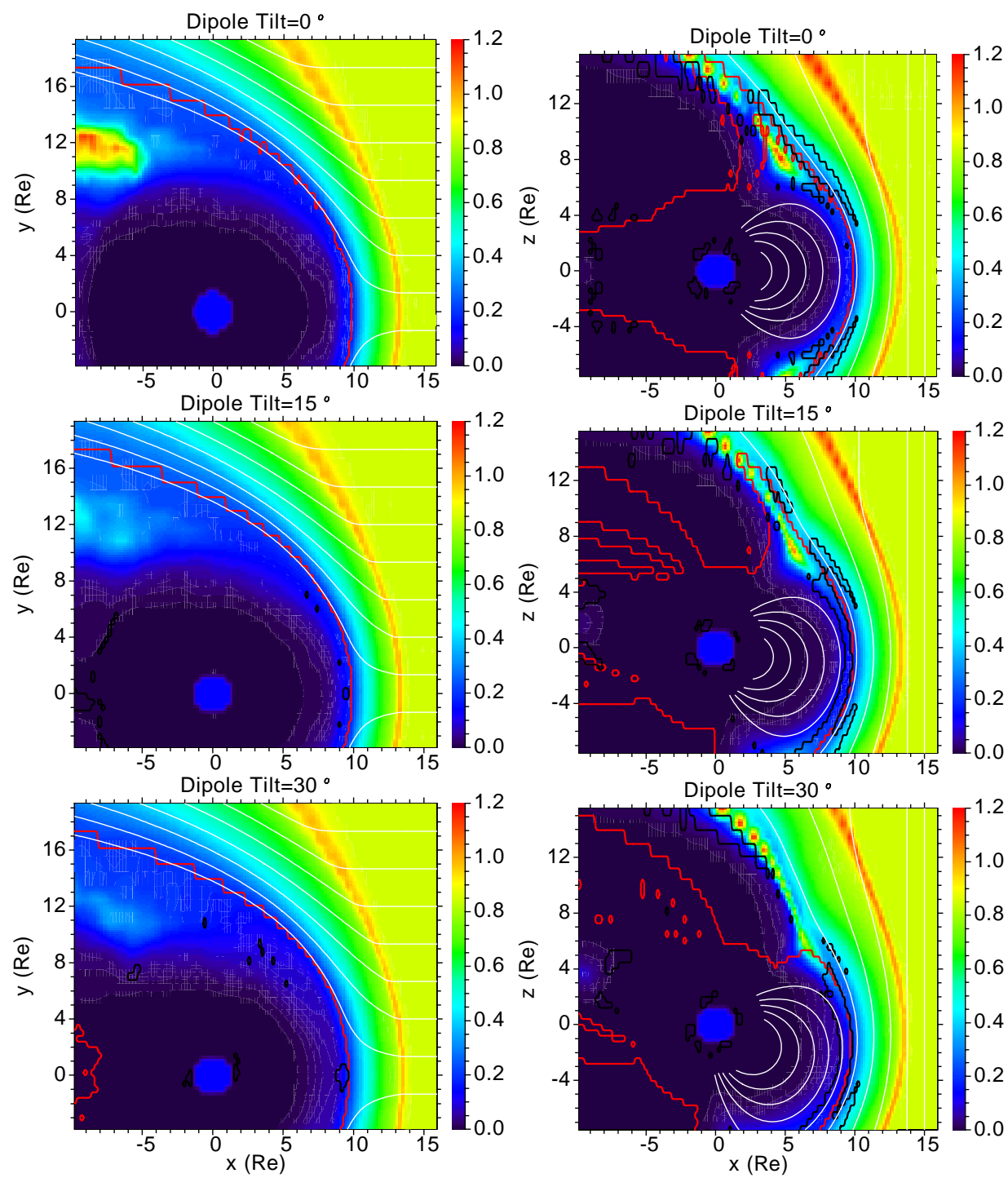

Fig. 13. The dependence of the PDL and the slow mode front on the Earth dipole tilt in the GSE $y=0$ plane: $0^{\circ}, 15^{\circ}$, and $30^{\circ}$. The baseline solar wind parameters are used as model inputs: $\boldsymbol{V}=(-450,0,0) \mathrm{km} / \mathrm{s}, \boldsymbol{B}=(0,0,7) \mathrm{nT}, \mathrm{N}=6 \mathrm{~cm}^{-3}$, and $\mathrm{T}_{\mathrm{p}}=\mathrm{T}_{\mathrm{e}}=10 \mathrm{eV}$. The other formats of this figure are the same as those in Fig. 3.

Table 6. The dependence of some important parameters along the Sun-Earth line on the Earth dipole tilt.

\begin{tabular}{ccccccccc}
\hline Dipole tilt $\left(^{\circ}\right)$ & $\mathrm{M}_{\mathrm{A}}$ & $\mathrm{M}_{\mathrm{MS}}$ & $\beta$ & $\lambda$ & $\mathrm{H}_{\mathrm{PDL}}\left(R_{E}\right)$ & $\mathrm{L}_{\mathrm{MP}}\left(R_{E}\right)$ & $\mathrm{L}_{\mathrm{BS}}\left(R_{E}\right)$ & $\mathrm{H}_{\mathrm{MS}}\left(R_{E}\right)$ \\
\hline 0 & 7.2 & 5.3 & 1.0 & 1.95 & 0.57 & 9.7 & 13.1 & 3.4 \\
15 & 7.2 & 5.3 & 1.0 & 1.41 & 0.60 & 10.2 & 13.2 & 3.0 \\
30 & 7.2 & 5.3 & 1.0 & 1.28 & 0.55 & 10.2 & 13.4 & 3.2 \\
\hline
\end{tabular}

We first introduce a new method to define the outer boundary of the plasma depletion layer. We believe that the ratio between the plasma density and the magnetic field magnitude (N/B) can give the best description of flux tube depletion in the magnetosheath, which is crucial for the formation of the PDL. In most of the cases, there are clear N/B ratio trend changes near the magnetopause, where we can define the outer boundary of the PDL. In the rare cases, where no clear
$\mathrm{N} / \mathrm{B}$ ratio trend change exists, we suggest using the density trend change as a replacement. Such a method is still different from the depletion factor method which uses a particular density ratio threshold instead of a density trend change to define the outer boundary of the PDL.

In the later part of the paper, we studied the dependence of the plasma depletion layer and the slow mode front on solar wind conditions and the Earth dipole tilt. We have 


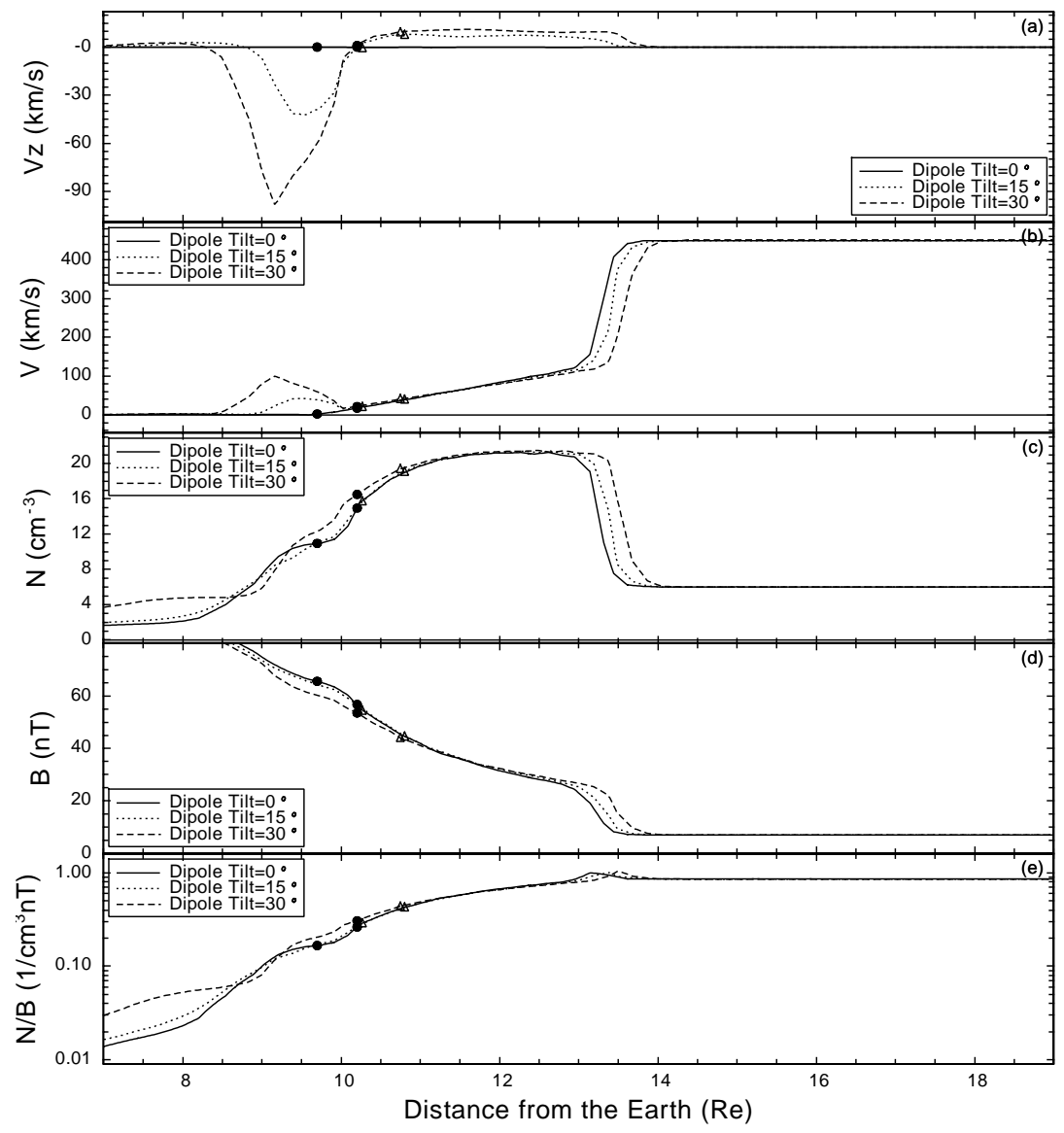

Fig. 14. The plasma $V_{z}$, the flow speed, the plasma density, the magnetic field magnitude, and the N/B ratio along the Sun-Earth line in Fig. 13. The black dot on each line marks the location of the magnetopause on that line which is defined by the close to zero flow velocity or the reverse of $V_{z}$. The other formats of this figure are the same as those in Fig. 4. found consistency and inconsistency between some of our results and former results from other people. For example, our results for different locations of the magnetopause using different definitions (stagnation point versus last closed magnetic field line) are consistent with the results of Song et al. (1999) and Siscoe et al. (2002). We also compared the plasma depletion factors from our study and some former studies for similar solar wind conditions (Zwan and Wolf, 1976; Wu, 1992; Lyon, 1994; Siscoe et al., 2002) and found that our results are intermediate among these results. In our study of the dependence of the PDL on the IMF tilt angle, we compared our results with the results of Lee et al. (1991) and found important differences between them. Specifically, Lee et al. (1991) found strong dependence of the enhanced plasma pressure and decreased magnetic field magnitude on the IMF $B_{x}$. While in our study, we found no such dependence. Actually, we do not see such structure for both IMF $B_{x}=0$ and $B_{x} \neq 0$. Using the ISM model, Siscoe et al. (2002) found a strong dependence of the PDL thickness on the IMF clock angle. In our study, however, a different trend and magnitude for such a dependence are obtained. The consistencies between our results and the former results tend to verify a certain understanding of some phenomena, while inconsistencies show the difficulties in some, if not all, models. We backup our simulation results by an earlier event study with satisfactory consistency between model PDL results and in- situ satellite PDL observations (Wang et al., 2003). On the other hand, most model studies compared in this paper used, at most, qualitative observation results. It is understandable that model verifications using realistic observations is a very difficult task for model studies, especially for those earlier ones. However, it has become more and more important for models to take this step of validation, which is crucial for applying the models in practice, including space weather prediction.

In almost all the cases of this study, we find strong dependence of the slow mode front on different solar wind conditions and the Earth dipole tilt. As discussed in detail in Wang et al. (2004b), such slow mode fronts cannot modify the flow and field structures in the magnetosheath in a significant way for the stable solar wind conditions in their study. However, it is still possible that those slow mode fronts can develop into large spatial structures, even shocks, during turbulent solar wind conditions. If this is the case, these slow mode front locations should be the points where we should check for such structures. And we predict a very strong dependence of the locations of these structures on many solar wind conditions and the Earth dipole tilt.

There have been many important features of the PDL and the slow mode front omitted in this study, including the extension of the PDL and the slow mode front in three dimensions, as well as the dependence of the PDL and the slow 
mode front on many other solar wind conditions. We have only concentrated on some of the most direct aspects of the PDL and the slow mode front dependence in this study. However, we have developed the most important techniques used for such a study, which makes it easier for future studies to better understand the PDL, and thus the coupling between the solar wind and the magnetosphere.

The major conclusions of this study are listed below:

1. There are difficulties for both the depletion factor method and the plasma $\beta$ method to define the outer boundary of the plasma depletion layer. The N/B ratio gives the best description of flux tube depletion, and its trend is used in this study for the definition of the PDL outer boundary. In the case when the N/B trend is not clear in the magnetosheath, the plasma density trend can be used as a complement.

2. The magnetosheath, including the PDL and the slow mode front, has a strong dependence on the solar wind magnetosonic Mach number. A difference between the magnetopause, defined as the stagnation point, and the magnetopause, defined as the open-closed magnetic field boundary, is found from our results, which is consistent with the results of Song et al. (1999) and Siscoe et al. (2002).

3. There is a strong dependence of the PDL and the slow mode front on the IMF $B_{z}$. For the IMF $B_{z}$ values used in the tests, the locations of the magnetopause and the bow shock, and the magnetosheath thickness change almost linearly. However, the plasma depletion factor first increases then decreases with the IMF $B_{z}$. A density structure is seen inside the subsolar magnetopause, which might be caused by the polar reconnection. The depletion factor is greatly affected by this density structure, thus we should be cautious in understanding this result. Further, different solar wind magnetosonic Mach numbers correspond to different IMF $B_{z}$. Thus, solar wind magnetosonic Mach number effects are not removed from this study, which requires more caution in understanding the results.

4. The IMF tilt angle leads to N/B asymmetry in the magnetosheath. However, it does not change the locations of the magnetopause and the bow shock along the SunEarth line in a significant way. The PDL is also only slightly influenced by the IMF tilt angle and the IMF $B_{x}$ does not seem to have a major influence on the PDL. This result is different from the results by Lee et al. (1991). The slow mode front has a much stronger dependence on the IMF tilt angle than the PDL.

5. The IMF clock angle is found to have little influence on the geometry of the magnetosheath for the solar wind inputs in this study. The PDL along the Sun-Earth line is only slightly influenced by the IMF clock angle, which is inconsistent with the global model results by Siscoe et al. (2002). Again, the slow mode front shows a strong dependence on the IMF clock angle.

6. The Earth dipole tilt does not change the magnetosheath geometry and the PDL along the Sun-Earth line in a significant way. However, it can change the depletion factor significantly. The slow mode front also shows complex geometry, which can easily extend to the GSE z=0 plane for a larger Earth dipole tilt.

Acknowledgements. The authors of the paper would like to thank M. G. Kivelson and R. J. Strangeway for their helpful discussions, as well as the two referees for their comments that are important to improve this paper. This work was supported by grant ATM-0084483 and grant ATM-01-01145 from United States National Science Foundation and grant NAG 5-10986 from the National Aeronautics and Space Administration. Computations were performed at San Diego Supercomputer Center. IGPP publication number 5838.

Topical Editor T. Pulkkinen thanks M. Witberger and another referee for their help in evaluating this paper.

\section{References}

Crooker, N. U., Eastman, T. E., and Stiles, G. S.: Observations of plasma depletion in the magnetosheath at the dayside magnetopause, J. Geophys. Res., 84, 869-874, 1979.

Denton, R. E. and Lyon, J. G.: Effect of pressure anisotropy on the structure of a two-dimensional magnetosheath, J. Geophys. Res., 105, 7545-7556, 2000.

Dungey, J. W.: Interplanetary magnetic field and the auroral zones, Phys. Rev. Lett., 6, 47-48, 1961.

Farrugia, C. J., Erkaev, N. V., Biernat, H. K., Lawrence, G. R., and Elphic, R. C.: Plasma depletion layer model for low Alfvén Mach number: Comparison with ISEE observations, J. Geophys. Res., 102, 11315-11 324, 1997.

Fuller-Rowell, T. J., Rees, D., Quegan, S., Moffett, R. J., Codrescu, M. V., and Millward, G. H.: A coupled thermosphere-ionosphere model (CTIM), in: STEP Report, edited by: Schunk, R., Scientific Committee on Solar Terrestrial Physics (SCOSTEP), NOAA/NGDC, Boulder, Colorado, 217-238, 1996.

Lee, L. C., Yan, M., and Hawkins, J. G.: A study of slow-mode structures in front of the dayside magnetopause, Geophys. Res. Lett., 18, 381-384, 1991.

Lyon, J. G.: MHD simulations of the magnetosheath, Adv. Space Res., 14, 21-28, 1994.

Paschmann, G., Baumjohann, W., Sckopke, N., Phan, T. D., and Lühr, H.: Structure of the dayside magnetopause for low magnetic shear, J. Geophys. Res., 98, 13 409-13 422, 1993.

Phan, T. D., Paschmann, G., Baumjohann, W., Sckopke, N., and Lühr, H.: The magnetosheath region adjacent to the dayside magnetopause: AMPTE/IRM observations, J. Geophys. Res., 99, 121-141, 1994.

Raeder, J.: Global magnetohydrodynamics - A tutorial, in: Space Plasma Simulation, edited by: Buchner, J., Dum, C. T., and Scholer, M., Springer Verlag, Heidelberg, Lecture Notes in Physics, Volume 615, 212-246, 2003.

Raeder, J., McPherron, R. L., Frank, L. A., Paterson, W. R., Sigwarth, J. B., Lu, G., Singer, H. J., Kokubun, S., Mukai, T., and Slavin, J. A.: Global simulation of the Geospace Environment Modeling substorm challenge event, J. Geophys. Res., 106, 381395, 2001a. 
Raeder, J., Wang, Y. L., and Fuller-Rowell, T. J.: Geomagnetic storm simulation with a coupled magnetosphere-ionospherethermosphere model, in: Space Weather, edited by: Song, P., Singer, H. J., and Siscoe, G. L., Geophysical Monograph Series, Volume 125, 377-384, 2001b.

Siscoe, G. L., Crooker, N. U., Erickson, G. M., Sonnerup, B. U. Ö., Maynard, N. C., Schoendorf, J. A., Siebert, K. D., Weimer, D. R., White, W. W., and Wilson, G. R.: MHD properties of magnetosheath flow, Planet. Space Sci., 50, 461-471, 2002.

Song, P. and Russell, C. T.: Model of the formation of the lowlatitude boundary layer for strongly northward interplanetary magnetic field, J. Geophys. Res., 97, 1411-1420, 1992.

Song, P. and Russell, C. T.: Flow in the magnetosheath: The legacy of John Spreiter, Planet. Space Sci., 50, 447-460, 2002.

Song, P., Elphic, R. C., Russell, C. T., Gosling, J. T., and Cattell, C. A.: Structure and properties of the subsolar magnetopause for northward IMF: ISEE observations, J. Geophys. Res., 95, 63756387, 1990a.

Song, P., Russell, C. T., Gosling, J. T., Thomsen, M., and Elphic, R. C.: Observations of the density profile in the magnetosheath near the stagnation streamline, Geophys. Res. Lett., 17, 20352038, 1990b.

Song, P., Russell, C. T., and Thomsen, M. F.: Slow mode transition in the frontside magnetosheath, J. Geophys. Res., 97, 8295$8305,1992$.
Song, P., Russell, C. T., Zhang, X. X., Stahara, S. S., Spreiter, J. R., and Gombosi, T. I.: On the process in the terrestrial magnetosheath, 2. Case study, J. Geophys. Res., 104, 22 357-22 373, 1999.

Southwood, D. J. and Kivelson, M. G.: On the form of the flow in the magnetosheath, J. Geophys. Res., 97, 2873-2879, 1992.

Southwood, D. J. and Kivelson, M. G.: Magnetosheath flow near the subsolar magnetopause: Zwan-Wolf and SouthwoodKivelson theories reconciled, Geophys. Res. Lett., 22, 32753278, 1995.

Spreiter, J. R., Summers, A. L., and Alksne, A. Y.: Hydromagnetic flow around the magnetosphere, Planet. Space Sci., 14, 223-253, 1966.

Wang, Y. L., Raeder, J., Russell, C. T., Phan, T. D., and Manapat, M.: Plasma depletion layer: Event studies with a global model, J. Geophys. Res., 108, 1010, doi:10.1029/2002JA009281, 2003.

Wang, Y. L., Raeder, J., and Russell, C. T.: Plasma depletion layer: Magnetosheath flow structure and forces, Ann. Geophys., 22, 1001-1017, 2004a.

Wang, Y. L., Raeder, J., and Russell, C. T.: Plasma depletion layer: The role of the slow mode waves, Ann. Geophys., 22, 42594272, 2004b.

Wu, C. C.: MHD flow past an obstacle: Large-scale flow in the magnetosheath, Geophys. Res. Lett., 19, 87-90, 1992.

Zwan, B. J. and Wolf, R. A.: Depletion of solar wind plasma near a planetary boundary, J. Geophys. Res., 81, 1636-1648, 1976. 